\title{
Fatores preditores da evasão escolar entre adolescentes com experiência de gravidez
}

\author{
School evasion predictive factors among adolescents with \\ pregnancy experience
}

\author{
Carolina Rodrigues de Oliveira Sousa ${ }^{1}$, Keila Rejane Oliveira Gomes ${ }^{1}$, \\ Kamila Cristiane de Oliveira Silva ${ }^{2}$, Márcio Dênis Medeiros Mascarenhas ${ }^{1}$, \\ Malvina Thaís Pacheco Rodrigues ${ }^{1}$, Jesusmar Ximenes Andrade ${ }^{1}$, \\ Maria Andréia Brito Ferreira Leal ${ }^{1}$
}

\begin{abstract}
Resumo
Introdução: A educação formal é considerada um fator protetor em razão da inclusão social que promove e porque a permanência dos jovens na escola reduz o risco da maternidade precoce, o que leva a associar a gestação adolescente à evasão escolar. Objetivo: Analisar os fatores preditores da evasão escolar entre adolescentes com experiência de gravidez em Teresina, Piauí. Método: Estudo transversal, com amostragem acidental, realizado com jovens que finalizaram uma gravidez no primeiro quadrimestre de 2006, quando tinham entre 15 e 19 anos de idade. Foi realizada análise multivariada por meio de regressão logística binomial para cálculo de Odds Ratio ajustado (ORaj), com intervalo de confiança de 95\% (IC 95\%). Resultados: 94,4\% das jovens afirmaram ter interrompido os estudos em algum momento da vida, das quais 54,4\% abandonaram os estudos. As jovens que trabalhavam e tiveram gravidez recorrente foram as mais propensas a abandonar os estudos. Além disso, conviver com uma renda f amiliar de até um salário mínimo aumentou suas chances em três vezes. O tempo em que parou de estudar se apresentou como fator de proteção. Conclusão: $O$ abando escolar de jovens mães está fortemente associado a fatores socioeconômicos desfavoráveis, por isso são imprescindíveis políticas que incentivem a continuidade dos estudos e, consequentemente, a inserção no mercado de trabalho, favorecendo melhor renda, menor dependência financeira, menos gravidez precoce e não planejadas.
\end{abstract}

Palavras-chave: gravidez; adolescência; escolaridade.

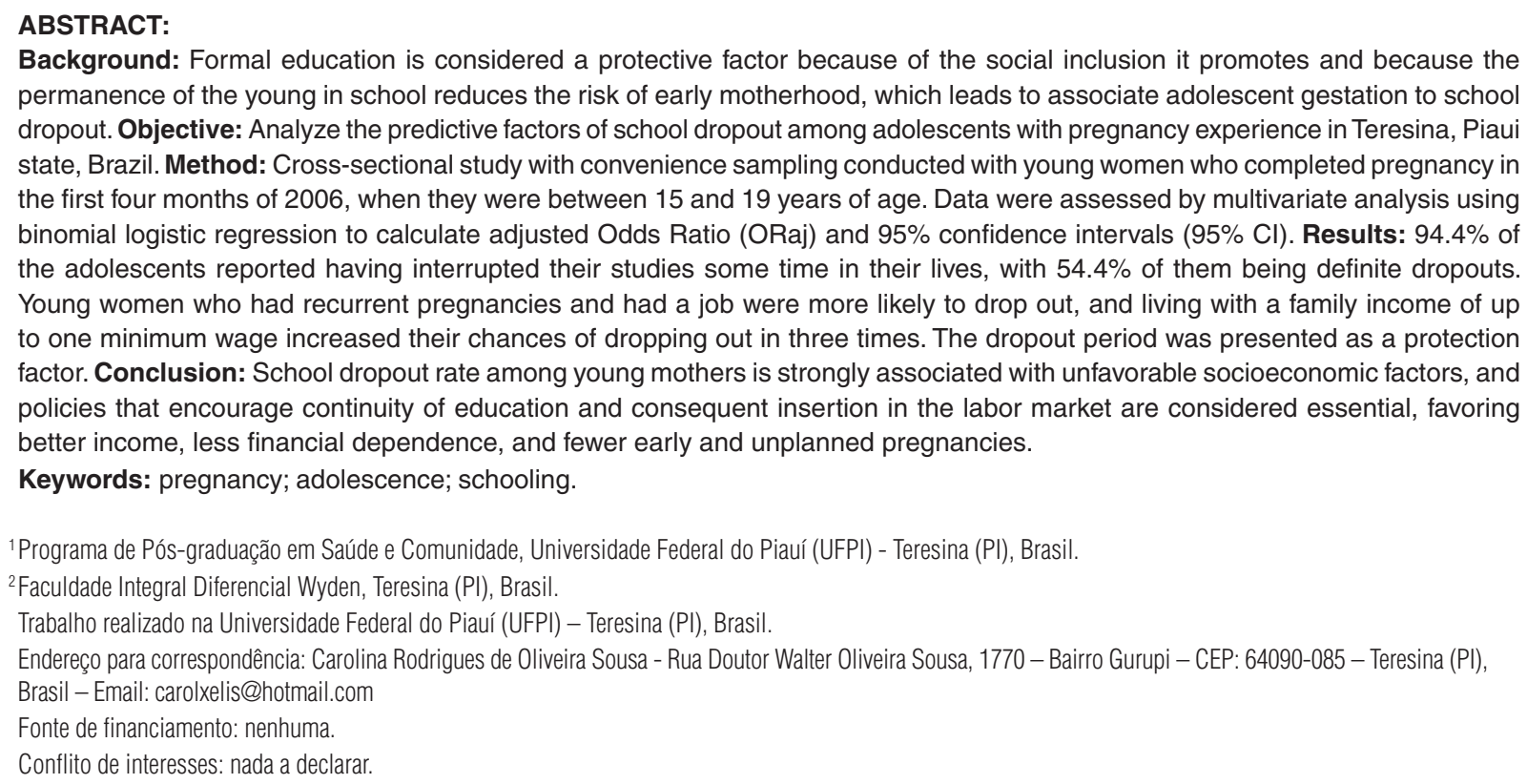
permanence of the young in school reduces the risk of early motherhood, which leads to associate adolescent gestation to school dropout. Objective: Analyze the predictive factors of school dropout among adolescents with pregnancy experience in Teresina, Piaui state, Brazil. Method: Cross-sectional study with convenience sampling conducted with young women who completed pregnancy in the first four months of 2006, when they were between 15 and 19 years of age. Data were assessed by multivariate analysis using binomial logistic regression to calculate adjusted Odds Ratio (ORaj) and 95\% confidence intervals (95\% Cl). Results: $94.4 \%$ of the adolescents reported having interrupted their studies some time in their lives, with $54.4 \%$ of them being definite dropouts. Young women who had recurrent pregnancies and had a job were more likely to drop out, and living with a family income of up to one minimum wage increased their chances of dropping out in three times. The dropout period was presented as a protection factor. Conclusion: School dropout rate among young mothers is strongly associated with unfavorable socioeconomic factors, and policies that encourage continuity of education and consequent insertion in the labor market are considered essential, favoring better income, less financial dependence, and fewer early and unplanned pregnancies.

Keywords: pregnancy; adolescence; schooling.

${ }^{1}$ Programa de Pós-graduação em Saúde e Comunidade, Universidade Federal do Piauí (UFPI) - Teresina (PI), Brasil.

${ }^{2}$ Faculdade Integral Diferencial Wyden, Teresina (PI), Brasil.

Trabalho realizado na Universidade Federal do Piauí (UFPI) - Teresina (PI), Brasil.

Endereço para correspondência: Carolina Rodrigues de Oliveira Sousa - Rua Doutor Walter Oliveira Sousa, 1770 - Bairro Gurupi - CEP: 64090-085 - Teresina (PI), Brasil-Email: carolxelis@hotmail.com

Fonte de financiamento: nenhuma.

Conflito de interesses: nada a declarar.

Este é um artigo publicado em acesso aberto (Open Access) sob a licença Creative Commons Attribution, que permite uso, distribuição e reprodução em qualquer meio, sem restrições desde que o trabalho original seja corretamente citado. 


\section{INTRODUÇÃO}

A adolescência, definida pela Organização Mundial da Saúde (OMS) como o período de 10 a 19 anos de idade, é caracterizada pela transição da infância para a fase adulta e assinalada por intensas transformações físicas, por conflitos relacionados às incertezas, pela formação da identidade e da autoestima, pelas instabilidades familiares e sociais e pelo conhecimento da imagem corporal. É nessa fase que a sexualidade aflora, que muitos indivíduos iniciam sua vida sexual e que a ocorrência de uma gravidez pode gerar dúvidas e sentimentos de fragilidade no contexto individual e familiar ${ }^{1-3}$.

Em 2014, a média global da taxa de nascimentos entre mães de 15 a 19 anos foi de 49 por 1.000 adolescentes, estando as maiores taxas em países da África Subsaariana, Ásia, América Latina e Caribe. Em 2008, ano de realização do presente estudo, a taxa de partos nessa faixa etária foi em torno de $11 \%$, com 95\% dos casos incidindo em países de baixa e média renda, entre os quais constavam a gravidez e o parto não planejados ou desejados. Nesse mesmo ano, a proporção no Brasil foi de 19,4\%, com decréscimo para 18,3\% em 2012; já no Piauí, adolescentes de 15 a 19 anos deram à luz a 22,9\% de todos os partos, com decréscimo para 20,7\% em 2012 $2^{4-7}$.

Apesar da queda nas taxas nacionais, por sua multidimensionalidade, a gravidez na adolescência permanece como uma questão preocupante para a saúde pública, uma vez que pode interromper importantes estágios de maturação psicossexual e provocar desorganização familiar, abandono escolar, afastamento social e do mercado de trabalho, aumento dos índices de pobreza e, dessa forma, gerar vários desafios para a equipe de saúde, educadores, governos e sociedade em geral ${ }^{8,9}$.

Das consequências sociais da gravidez na adolescência que mais têm sido debatidas na literatura estão aquelas relacionadas à escolarização. $\mathrm{O}$ ambiente no qual esses jovens estão inseridos é responsável pela formação e pelo controle de suas vidas, e a educação constitui fator importante para o sucesso desse processo ${ }^{4}$.

A educação formal é considerada um fator protetor em razão da inclusão social que promove e porque a permanência dos jovens na escola reduz o risco da maternidade precoce, $o$ que leva a associar a gestação adolescente à evasão escolar ${ }^{10,11}$. Em 2014, cerca de 1/3 das jovens brasileiras de 15 a 17 anos que abandonaram a escola já era mãe, e, daquelas que estudavam, apenas $2 \%$ tinham filho ${ }^{12}$. Essa é uma realidade não só no Brasil, mas na América Latina, visto que, em 2016, as mulheres latinas representaram $2 / 3$ das jovens de 15 a 17 anos que não estudavam nem trabalhavam, com a gravidez na adolescência sendo apontada como uns dos principais fatores de risco para o abandono escolar ${ }^{13}$.

É o ensino que dá ao indivíduo a capacidade de atuar na sua realidade e exercer sua cidadania, de ter um futuro promissor e, no que diz respeito à sexualidade, pode influenciar o risco de gravidez por meio do desenvolvimento sociocognitivo, do capital humano ou da exposição a diferentes redes sociais e sexuais. Portanto, a sua interrupção, quando de uma gestação precoce e, principalmente, em adolescentes de classes menos favorecidas da sociedade, coloca essas jovens em desvantagem para o alcance de melhores postos de trabalho e contribui para a perpetuação da pobreza, conforme vários estudos ${ }^{3,8,14}$.

Por outro lado, também tem sido mostrado que a pobreza na infância aumenta as chances de evasão e/ou repetência escolar, e a consequente falta de oportunidades e as desigualdades sociais levam as adolescentes a engravidar precocemente. Tal situação revela os diferentes desdobramentos que a gravidez na adolescência tem para grupos sociais diversos: enquanto mães adolescentes oriundas de famílias de baixa renda ficam fora da escola e dedicam-se ao papel de donas de casa, as filhas da classe média não desorganizam suas trajetórias acadêmicas, mesmo quando um atraso é possível ${ }^{2,10,15}$.

Portanto, como há controvérsia sobre a gravidez precoce ser causa ou consequência do abandono escolar, o presente estudo objetiva analisar os fatores preditores da evasão escolar entre adolescentes com experiência de gravidez no contexto de baixa renda familiar, a fim de identificar se as jovens haviam abandonado a escola antes de engravidarem ou se a gravidez foi prévia ao abandono escolar. Dessa forma, espera-se contribuir para o desenvolvimento de políticas públicas direcionadas à valorização e ao atendimento às demandas de jovens, tais como essa problemática da gravidez precoce e do abandono escolar.

\section{MÉTODO}

Trata-se de estudo transversal, parte de projeto de pesquisa intitulado "Gravidez na adolescência: fatores preditores da reincidência", que, em 2008, teve dados coletados de adolescentes que finalizaram uma gravidez no primeiro quadrimestre de 2006, período em que tinham de 15 a 19 anos de idade. As instituições participantes foram seis maternidades de Teresina, Piauí, das quais cinco eram públicas e uma era privada.

A amostragem acidental, que consiste na coleta de dados dos casos ocorridos em determinado período de tempo, foi a opção escolhida devido à impossibilidade do cálculo da amostra probabilística, uma vez que, diante da limitação de não se identificarem todos os tipos de resolução de gravidez, como é o caso do abortamento, todas as formas seriam incluídas. Como o método de localização das mulheres foi o da busca ativa dois anos após o parto, optou-se pelo acesso aos registros hospitalares para obtenção de endereços e números de telefone para o contato com as potenciais adolescentes eleitas para o estudo. Ao final, foram localizados 632 casos.

Após a localização dos casos a partir dos registros das maternidades, as entrevistadoras realizavam contato telefônico 
ou encaminhavam-se aos domicílios das jovens convidando-as a participar do estudo. Aquelas adolescentes não localizadas pelas entrevistadoras eram repassadas para supervisora de campo, a fim de tentar localizar o endereço com a ajuda dos agentes comunitários de saúde das equipes da Estratégia Saúde da Família, tanto da área de localização inicial quanto da área para a qual ela supostamente havia se mudado, cujo bairro de destino era fornecido por vizinhos. Caso a jovem tivesse se mudado para outra cidade ou quando toda estratégia de localização não obtinha êxito, o caso foi considerado perdido.

Considerou-se como desfecho a variável evasão escolar (variável dependente). Como variáveis independentes, selecionaram-se as relativas aos dados sociodemográficos, à história obstétrica e à trajetória escolar. Os dados originais foram digitados em dois bancos de dados no aplicativo Epi Info, versão 6.04d (U.S Center for Disease Control and Prevention, Atlanta, GA). A análise estatística foi feita pelo programa Statistical Package for the Social Sciences, versão 17.0 (SPSS Inc. Chicago, IL 60606, USA).

Utilizou-se estatística descritiva para a análise univariada dos dados. Na análise bivariada, foi utilizada regressão logística simples (RLS) para identificar possíveis associações entre cada variável independente (sociodemográficas, história obstétrica e trajetória escolar) e evasão escolar (variável dependente). A possível associação entre as varáveis foi determinada pelo Odds Ratio não ajustado (ORñaj), as quais, quando significantes, foram selecionadas para inclusão nos modelos desenvolvidos para a regressão logística múltipla (RLM).

Para a análise multivariada, usou-se regressão logística binomial para determinar possíveis fatores que pudessem ter levado à evasão escolar das mães adolescentes. As variáveis foram incluídas no modelo pelo método Enter, que força a entrada das variáveis de forma sequencial e isolada. Utilizou-se o Odds Ratio ajustado (ORaj) como medida de efeito, com intervalo de confiança de 95\% (IC95\%), e a hipótese de nulidade foi aceita sempre que o valor de p era maior ou igual a 0,05.

$\mathrm{O}$ teste de multicolinearidade necessário para a regressão logística binomial foi realizado pelo Variance inflation fator (VIF), adotando-se $\geq 4$ como ponto de corte; porém, não observou-se multicolinearidade entre as variáveis independentes.

O projeto-base, do qual faz parte este estudo, foi submetido ao Comitê de Ética em Pesquisa da Universidade Federal do Piauí (UFPI), sob CAAE n 0056.0.045.000-08, e aprovado, cumprindo todos os preceitos éticos e legais das pesquisas que envolvem seres humanos, conforme a Resolução nº 196/96 do Conselho Nacional de Saúde. Foi solicitada a assinatura do Termo de Consentimento Livre e Esclarecido (TCLE) para todas as participantes. Para jovens menores de 18 anos, a assinatura do TCLE foi solicita ao seu responsável.

\section{RESULTADOS}

A maioria das jovens, no momento da pesquisa, pertencia à faixa etária de 20 a 22 anos (69,8\%), com média de idade de 20,2 anos (desvio-padrão = 1,3). Em relação ao estado civil, 65,5\% eram casadas/em união estável, das quais quase que a totalidade referiu morar com o companheiro (63,6\%). Constatou-se que pouco mais de $72 \%$ das jovens não trabalhavam, metade vivia em famílias que ganhavam no máximo um salário mínimo vigente à época da coleta de dados e $45 \%$ moravam com famílias compostas de 5 a 10 pessoas. Aproximadamente $65 \%$ das jovens eram totalmente dependentes financeiramente de terceiros, das quais $50,4 \%$ tinham o companheiro como responsável pelo seu sustento, e 1 a cada 4 jovens tinha dependência financeira dos pais (Tabela 1).

Parcela significativa referiu que não frequentava uma unidade de ensino $(69,6 \%)$ e que parou os estudos em algum momento da vida $(94,4 \%)$ durante ou após a gravidez, a qual foi o principal motivo para quase metade delas. Após a interrupção, que ocorreu predominantemente durante a primeira gestação $(49,2 \%)$, $59 \%$ não mais retornaram à escola, das quais 55,2\% alegaram como motivo o cuidar da família e da casa. As jovens que continuaram a frequentar uma unidade de ensino perceberam, por parte das pessoas da escola, mais aceitação (86\%) do que críticas (14\%), as quais ocorreram em maior parte por outros estudantes (70,8\%). O nível de escolaridade mais frequente entre as jovens foi o ensino fundamental incompleto $(34,7 \%)$, semelhante ao de suas mães (38,5\%), além de que a maioria não tinha adequação com a idade (86,9\%). De maneira expressiva, $55,4 \%$ mencionaram abandono escolar (Tabela 2).

No que diz respeito à história reprodutiva, $41,8 \%$ das participantes tiveram a primeira gestação na faixa etária de 16 a 17 anos, com média de 1,8 gestação (desvio-padrão $=1,0$ ). Em torno de $50 \%$ eram primigestas, e 1 a cada 5 já havia engravidado pelo menos três vezes. Ademais, $63 \%$ eram filhas de mãe que engravidaram na adolescência e 48,9\% tinham pelo menos um irmão ou uma irmã que também teve filho na adolescência (Tabela 3).

A Tabela 4 exibe os modelos de análise multivariada. Os modelos 1, 2 e 3 incluem, respectivamente, a experiência de gravidez da participante, as variáveis relativas à sua escolaridade e de sua mãe e as suas características sociodemográficas. O modelo 4 é o completo, por incluir todas as variáveis que tenham sido significativas nos modelos anteriores.

A variável "momento em que parou de estudar com relação às gestações" mostrou-se significativa nos modelos $1,3 \mathrm{e} 4 \mathrm{e}$ indicou que a chance de abandono escolar foi maior depois de as adolescentes engravidarem pela primeira vez. As razões de chance entre os modelos não apresentaram variações importantes com a inclusão de outros fatores. 
Tabela 1. Características sociodemográficas de jovens com história de gravidez em Teresina, Piauí, 2008

\begin{tabular}{|c|c|c|c|}
\hline Variáveis & $\overline{\boldsymbol{x}} \mathrm{i}(s) \mathrm{ii}$ & $\mathbf{n}$ & $\%$ \\
\hline Faixa etária (anos) $)^{\mathrm{a}}$ & $20.2(1.3)$ & 464 & 100,0 \\
\hline $17-19$ & & 140 & 30,2 \\
\hline $20-22$ & & 324 & 69,8 \\
\hline Estado civil ${ }^{\mathrm{b}}$ & & 464 & 100,0 \\
\hline Solteira & & 160 & 34,5 \\
\hline Casada/em união estável & & 304 & 65,5 \\
\hline Mora com o companheiro & & 464 & 100,0 \\
\hline Sim & & 295 & 63,6 \\
\hline Não, mas mantém os laços conjugais & & 61 & 13,1 \\
\hline Não, e não mantém os laços conjugais & & 108 & 23,3 \\
\hline Trabalho remunerado & & 464 & 100,0 \\
\hline Sim & & 129 & 27,8 \\
\hline Não & & 335 & 72,2 \\
\hline Renda familiar & & 464 & 100,0 \\
\hline Até 1/2 salário mínimo (SM) (até R\$206,00) & & 60 & 12,9 \\
\hline Até $1 \mathrm{SM}(\mathrm{R} \$ 206,01$ a $\mathrm{R} \$ 412,00)$ & & 172 & 37,1 \\
\hline Entre 1 e 2 SM (R\$ 412,01 a R\$824,00) & & 135 & 29,1 \\
\hline Entre 2 a 3 SM $(\mathrm{R} \$ 824,01$ a $\mathrm{R} \$ 1.236,00)$ & & 48 & 10,3 \\
\hline Mais de $3 \mathrm{SM}$ ( $\mathrm{R} \$ 1.236,01$ ou mais) & & 49 & 10,6 \\
\hline Com quantas pessoas mora em casa ${ }^{c}$ & $5.1(2.5)$ & 464 & 100,0 \\
\hline 1 a 4 pessoas & & 243 & 52,4 \\
\hline 5 a 10 pessoas & & 208 & 44,8 \\
\hline Mais de 10 pessoas & & 13 & 2,8 \\
\hline Dependência financeira & & 464 & 100,0 \\
\hline Sim, parcialmente & & 101 & 21,8 \\
\hline Sim, totalmente & & 300 & 64,7 \\
\hline Não & & 63 & 13,6 \\
\hline De quem depende financeiramente & & 401 & 100,0 \\
\hline Companheiro & & 202 & 50,4 \\
\hline Pais dela & & 111 & 27,7 \\
\hline Pais dele (companheiro) & & 5 & 1,2 \\
\hline Qualquer membro da família dele/dela & & 14 & 3,5 \\
\hline Outra(s) pessoa(s) & & 5 & 1,2 \\
\hline Mais de uma das opções anteriores & & 64 & 16,0 \\
\hline
\end{tabular}

Tabela 2. Características da escolaridade de jovens com história de gravidez em Teresina, Piauí, 2008

\begin{tabular}{lcr}
\multicolumn{1}{c}{ Variáveis } & n & \% \\
Ir à escola/à universidade/ao curso técnico, atualmente & $\mathbf{4 6 4}$ & $\mathbf{1 0 0 , 0}$ \\
Sim, escola/universidade/curso & 120 & 25,9 \\
Sim, curso técnico & 21 & 4,5 \\
Não & 323 & 69,6 \\
Parou de estudar em algum momento & $\mathbf{4 6 4}$ & $\mathbf{1 0 0 , 0}$ \\
Sim & 438 & 94,4 \\
Não & 26 & 5,6 \\
Razão para parar & $\mathbf{4 3 8}$ & $\mathbf{1 0 0 , 0}$ \\
Ficou grávida & 213 & 48,6 \\
Para cuidar da família/casa & 62 & 14,1 \\
Preguiça/não gosta de estudar/se divertir/não queria & 55 & 12,6 \\
Completou (ensino médio) & 44 & 10,0 \\
Trabalhar & 23 & 5,3 \\
Complicações na gravidez & 12 & 2,7 \\
Problemas com a escola & 6 & 1,4 \\
Outras razões & 23 & 5,3 \\
\hline
\end{tabular}


Tabela 2. Continuação...

\begin{tabular}{|c|c|c|}
\hline Variáveis & $\mathbf{n}$ & $\%$ \\
\hline Você voltou a estudar depois desse intervalo & 438 & 100,0 \\
\hline Sim & 181 & 41,3 \\
\hline Não & 257 & 58,7 \\
\hline Razão para não voltar à escola & 257 & 100,0 \\
\hline Para cuidar da família/casa & 142 & 55,2 \\
\hline Não quis & 43 & 16,7 \\
\hline Outras & 30 & 11,7 \\
\hline Por causa do trabalho & 21 & 8,2 \\
\hline Não tem tempo/paciência & 12 & 4,7 \\
\hline Terminou o ensino médio & 9 & 3,5 \\
\hline Quanto à gravidez, quando você parou de estudar & 429 & 100,0 \\
\hline Antes de engravidar pela $1^{\mathrm{a}} \mathrm{vez}$ & 107 & 24,9 \\
\hline Durante a $1^{\text {a }}$ gravidez & 211 & 49,2 \\
\hline Entre a $1^{\text {a }}$ e a $2^{\text {a }}$ gravidez & 21 & 4,9 \\
\hline Após a última gravidez & 65 & 15,2 \\
\hline Outra situação & 25 & 5,8 \\
\hline Como você foi recebida na escola após a gravidez & 344 & 100,0 \\
\hline Aceitaram & 296 & 86,0 \\
\hline Foi criticada & 48 & 14,0 \\
\hline Quem criticou você & 48 & 100,0 \\
\hline Professor(es) & 3 & 6,2 \\
\hline Empregado(s) & 2 & 4,2 \\
\hline Colega(s) de classe & 34 & 70,8 \\
\hline Pai(s) de aluno & 3 & 6,2 \\
\hline Mais de uma categoria anterior & 6 & 12,5 \\
\hline Escolaridade & 464 & 100,0 \\
\hline Analfabeta funcional ( $<4$ anos de estudo) & 6 & 1,3 \\
\hline Ensino fundamental incompleto ( $4 \leq \mathrm{x}<7$ anos de estudo) & 161 & 34,7 \\
\hline Ensino fundamental & 55 & 11,9 \\
\hline Ensino médio incompleto & 149 & 32,1 \\
\hline Ensino médio & 82 & 17,7 \\
\hline Ensino superior (incompleto/completo) & 11 & 2,4 \\
\hline Escolaridade da mãe das jovens & 429 & 100,0 \\
\hline Não alfabetizada & 66 & 14,3 \\
\hline Analfabeta funcional ( $<4$ anos de estudo) & 54 & 11,7 \\
\hline Ensino fundamental incompleto ( $4 \leq \mathrm{x}<7$ anos de estudo) & 178 & 38,5 \\
\hline Ensino fundamental & 36 & 7,8 \\
\hline Ensino médio incompleto & 35 & 7,6 \\
\hline Ensino médio & 48 & 10,4 \\
\hline Ensino superior (incompleto/completo) & 12 & 2,6 \\
\hline Não sabe & 33 & 7,1 \\
\hline Adequação idade-série & 464 & 100,0 \\
\hline Sim & 61 & 13,1 \\
\hline Não & 403 & 86,9 \\
\hline Abandonou a escola & 464 & 100,0 \\
\hline Sim & 257 & 55,4 \\
\hline Não & 207 & 44,6 \\
\hline
\end{tabular}


Tabela 3. História reprodutiva de jovens com história de gravidez em Teresina, Piauí, 2008

\begin{tabular}{|c|c|c|c|}
\hline Variáveis & $\overline{\boldsymbol{x}}_{\mathrm{i}}(s)_{\mathrm{ii}}$ & $\mathbf{n}$ & $\%$ \\
\hline Idade na $1^{\text {a }}$ gravidez & $16,6(1,6)$ & 464 & 100,0 \\
\hline $12-14$ & & 53 & 11,4 \\
\hline 15 & & 70 & 15,1 \\
\hline $16-17$ & & 194 & 41,8 \\
\hline $18-19$ & & 147 & 31,7 \\
\hline Quantas vezes engravidou & $1,8(1,0)$ & 464 & 100,0 \\
\hline 1 & & 228 & 49,1 \\
\hline 2 & & 146 & 31,5 \\
\hline$\geq 3$ & & 90 & 19,4 \\
\hline Idade com que a mãe da jovem engravidou pela primeira vez & $20,5(10,8)$ & 464 & 100,0 \\
\hline $11-19$ & & 285 & 62,8 \\
\hline 20 ou mais & & 169 & 37,2 \\
\hline Irmão ou irmã da jovem teve filho antes dos 20 anos de idade & & 451 & 100,0 \\
\hline Sim & & 217 & 48,9 \\
\hline Não & & 234 & 51,9 \\
\hline
\end{tabular}

Tabela 4. Odds ratio $(O R)$ ajustado da associação entre história reprodutiva, história escolar, características sociodemográficas, modelo completo e abandono escolar de jovens com história de gravidez em Teresina, Piauí, 2008

\begin{tabular}{|c|c|c|c|c|c|c|c|c|}
\hline \multirow{2}{*}{ Variáveis } & \multicolumn{2}{|c|}{ Modelo 1} & \multicolumn{2}{|c|}{ Modelo 2} & \multicolumn{2}{|c|}{ Modelo 3} & \multicolumn{2}{|c|}{ Modelo 4} \\
\hline & OR & CI $95 \%$ & OR & CI $95 \%$ & OR & CI $95 \%$ & OR & CI $95 \%$ \\
\hline \multirow{2}{*}{\multicolumn{9}{|c|}{$\begin{array}{l}\text { Tempo que você deixou de estudar em relação } \\
\text { à gravidez }\end{array}$}} \\
\hline & & & & & & & & \\
\hline \multicolumn{9}{|l|}{ Antes de engravidar pela $1^{\text {a }}$ vez $\left.{ }^{(* *}\right)$} \\
\hline Depois de engravidar pela $1^{a}$ vez & $0,55^{\star}$ & $0,34-0,88$ & 0,61 & $0,37-1,00$ & $0,55^{\star}$ & $0,33-0,94$ & $0,59^{*}$ & $0,36-0,96$ \\
\hline Quantas vezes você engravidou & $1,40^{*}$ & $1,13-1,74$ & - & - & - & - & $1,35^{\star}$ & $1,07-1,71$ \\
\hline \multicolumn{9}{|l|}{ Escolaridade das jovens } \\
\hline $\begin{array}{l}<7 \text { anos de estudo } \\
\left.\geq 7 \text { anos de estudo }{ }^{* *}\right)\end{array}$ & - & - & $1,87^{\star}$ & $1,20-2,93$ & - & - & 0,71 & $0,45-1,12$ \\
\hline Adequação idade-série & & & & & - & - & - & - \\
\hline $\operatorname{Sim}(* *)$ & & & & & - & - & - & - \\
\hline Não & - & - & 0,71 & $0,34-1,49$ & - & - & - & - \\
\hline \multicolumn{9}{|l|}{ Escolaridade das mães } \\
\hline $\begin{array}{l}<7 \text { anos de estudo } \\
\geq 7 \text { anos de estudo }\left(^{* *}\right)\end{array}$ & - & - & 1,02 & $0,61-1,69$ & - & - & - & - \\
\hline \multicolumn{9}{|l|}{ Estado civil } \\
\hline \multicolumn{9}{|l|}{ Solteira $\left({ }^{* *}\right)$} \\
\hline Casada/em um relacionamento estável & - & - & - & - & 1,95 & $0,95-4,00$ & - & - \\
\hline \multicolumn{9}{|l|}{ Trabalho } \\
\hline $\begin{array}{l}\text { Sim } \\
\left.\text { Não }{ }^{* *}\right)\end{array}$ & - & - & - & - & $2,41^{*}$ & $1,33-4,38$ & $2,54^{\star}$ & $1,54-4,09$ \\
\hline \multicolumn{9}{|l|}{ Renda familiar } \\
\hline Até $1 / 2$ salário mínimo (SM) (até R \$206,00) & - & - & - & - & $5,67^{\star}$ & $2,10-15,34$ & $3,18^{\star}$ & $1,28-7,86$ \\
\hline Até 1 SM (R\$206,01 a R\$ 412,00) & - & - & - & - & $3,25^{\star}$ & $1,40-7,53$ & 1,89 & $0,88-4,03$ \\
\hline Entre 1 e 2 SM ( $R \$ 412,01$ a $R \$ 824,00)$ & - & - & - & - & $2,35^{\star}$ & $1,01-5,48$ & 1,71 & $0,81-3,60$ \\
\hline $\begin{array}{l}\text { Entre } 2 \text { e } 3 \text { SM }(\mathrm{R} \$ 824,01 \text { a } \mathrm{R} \$ 1.236,00) \\
\text { Mais de } 3 \mathrm{SM}\left(\mathrm{R} \$ 1.236,01 \text { ou mais) }\left(^{* *}\right)\right.\end{array}$ & - & - & - & - & 1,77 & $0,64-4,92$ & 1,54 & $0,62-3,83$ \\
\hline \multicolumn{9}{|l|}{$\begin{array}{l}\text { Quantas pessoas moram na sua casa } \\
1 \text { a } 4 \text { pessoas }\left({ }^{* *}\right)\end{array}$} \\
\hline $5 \mathrm{ou}+$ pessoas & - & - & - & - & 1,19 & $0,73-1,95$ & - & - \\
\hline \multicolumn{9}{|l|}{ De quem depende financeiramente } \\
\hline Pais dela/dele & - & - & - & - & 0,72 & $0,33-1,55$ & - & - \\
\hline Outra(s) pessoas(s) & - & - & - & - & 1,28 & $0,40-4,16$ & - & - \\
\hline Mais de uma opção anterior & - & - & - & - & 1,39 & $0,70-2,73$ & - & - \\
\hline
\end{tabular}

${ }^{\star} \mathrm{p} \leq 0,05 ;{ }^{* *}$ Categoria de referência. 
Quanto à variável "quantas vezes ficou grávida", a análise mostrou que, a cada gravidez, a chance de evasão escolar foi aumentada em $40 \%$ no modelo 1 e em $35 \%$ no modelo 4 . Os resultados dos modelos 3 e 4 apontaram que as adolescentes que trabalhavam foram mais propensas à evasão escolar que aquelas que não trabalhavam, sem alterações expressivas na razão de chances. A propensão das menos escolarizadas de evadirem-se da escola detectada no modelo 2 não se confirmou quando foram incluídas outras variáveis no modelo 4.

Observa-se, no modelo 3, que as chances de evasão escolar aumentaram à medida que se reduzia a renda familiar. Entretanto, o modelo 4 mostra que apenas as jovens com renda familiar de até $1 / 2$ salário mínimo tiveram a chance de abandono da escola aumentada quando comparadas àquelas com renda familiar de mais de três salários mínimos.

\section{DISCUSSÃO}

Observando o perfil das adolescentes entrevistadas, percebe-se uma concordância com outros estudos nacionais e internacionais ${ }^{16-20}$. Como o principal critério de inclusão do estudo foi que jovens tivessem uma resolução de gravidez no primeiro quadrimestre de 2006, momento em que elas ainda eram adolescentes com idade de 15 a 19 anos, a maioria das participantes já não era mais adolescente, mas permanecia no grupo dos considerados jovens pela Organização Mundial da Saúde ${ }^{21}$.

Diferente de países economicamente desfavoráveis, nos quais a união precoce é desejável em virtude do peso financeiro, no Brasil, classificado como país de renda média alta, casar precocemente pode ser a solução para jovens sexualmente ativas ou mesmo grávidas, para que não se tornem motivo de estigmas e, consequentemente, de constrangimentos aos pais e à família, ou ainda como saída para as faltas de oportunidades e uma alternativa para assegurar seu futuro financeiro ${ }^{3,22}$. Assim, o elevado número de uniões consensuais observado nesta pesquisa pode ser explicado pela pressão social para que o casal logo formalize a união diante de uma gravidez precoce ${ }^{16}$.

Nesse contexto, a dependência de terceiros e a construção de vários núcleos familiares em um mesmo espaço físico surgem como aspectos negativos, uma vez que, além de inserirem a jovem em situação de baixa renda, como demonstrado pelos resultados da pesquisa, tornam-na mais susceptível à violência física e de outras naturezas, tanto por parte do parceiro como de outros membros da família ${ }^{16,23}$.

As repercussões da gravidez na adolescência escolar têm sido objeto de estudo de diversos autores ${ }^{8,24-26}$. Os dados da presente pesquisa, bem como os do estudo realizado em municípios do Estado de São Paulo sobre gravidez na adolescência, que verificou que o abandono escolar referido por parcela significativa das moças estava relacionado à gravidez, além do estudo sobre indicadores sociais em uma população de gestantes, o qual estimou que apenas $6,8 \%$ delas eram estudantes, comprovam que a gestação muda a perspectiva da escolaridade, levando as adolescentes a parar de estudar temporária ou definitivamente ${ }^{2,27,28}$.

Contudo, se a gravidez na adolescência pode ocasionar a evasão escolar, alguns estudos, igualmente ao encontrado na presente pesquisa, têm provado que muitas adolescentes podem ter deixado a escola antes de engravidar, sendo inclusive uma condição de risco para gravidez precoce ${ }^{15,29}$. Isso pode ser justificado pelo fato de a gravidez nas adolescentes ter ocorrido já com elas em defasagem de idade-série, sendo um fator sobreposto à provável evasão escolar, o que parece indicar que, assim como o casamento, a maternidade se sobrepõe aos projetos educacionais e profissionais, uma vez que essa nova perspectiva de vida proporciona reconhecimento social ou, mais provavelmente e segundo a OMS, reflete a influência do contexto de pobreza no comportamento e nas escolhas das adolescentes ${ }^{3,30,31}$.

A baixa escolaridade confere ao indivíduo menor probabilidade de inserção no mercado de trabalho, cada vez mais competitivo. A adolescência é uma fase em que os indivíduos estão em formação, incluindo a escolar, e obtendo habilidades para terem maior chance de sucesso na vida adulta; portanto, assumir os papéis de mãe e de dona de casa diminui as possibilidades de qualificação profissional, prejudicando seu potencial produtivo e perpetuando a desvantagem social ${ }^{8,9,23}$.

Os resultados deste estudo mostraram que, apesar de a maioria das adolescentes, na análise univariada, ter informado não estar trabalhando, a associação entre a trajetória escolar e a história reprodutiva demonstrou que a baixa escolaridade e o número de gestações reforçam a evasão escolar das que trabalham. Algumas mães jovens descrevem suas atividades profissionais como necessárias para cumprir seu projeto de vida, mesmo que afirmem o conflito que o trabalho gera nas funções da maternidade, e de baixa remuneração. Além disso, é a inserção nessas primeiras atividades que faz com que essas adolescentes saiam da escola ou a frequentem irregularmente, prejudicando sua realização escolar ${ }^{9,15}$.

A recorrência da gravidez também esteve associada ao abandono escolar. Variáveis relacionadas com o desempenho escolar têm sido fortemente associadas à ocorrência de gravidez na adolescência, de gestações sucessivas e de gestações de rápida repetição nessa fase da vida ${ }^{32-34}$. Estudo realizado na África do Sul com jovens da zona rural mostrou que estar na escola constitui fator protetor para a gravidez na adolescência, assim como para sua repetição. Além disso, estudo comparativo entre primíparas e multíparas evidenciou menor escolaridade e maior abandono dos estudos entre as segundas ${ }^{14}$.

Abandonar os estudos por conta de uma gravidez precoce traz graves consequências para adolescente e sua prole, uma 
vez que a principal forma de ascensão social e econômica se dá por meio do sistema educacional, e limita as possibilidades em conseguir trabalhos mais bem remunerados por causa de que estes exigem habilidades que dificilmente são alcançáveis por populações economicamente desfavoráveis ${ }^{3,19,33}$. Visto que é pouco provável o retorno aos estudos conforme demonstrado nesta pesquisa, conviver com a pobreza passa a ser a realidade da maioria e perpetuado por sua prole, algo bastante verificado na literatura e que foi comprovado por esta amostra, na qual a maioria das adolescentes referiu casos de gravidez na adolescência de irmão ou irmã, cuja mãe também engravidou na adolescência ${ }^{2,23,31}$.

A situação econômica, traduzida pela renda familiar, de maneira consequente também está associada à interrupção dos estudos. Esse resultado obtido nesta pesquisa evidencia que jovens provenientes de segmentos com níveis de renda inferiores enfrentam barreiras que dificultam o prosseguimento de trajetórias ascendentes e de qualidade ${ }^{2,17,19}$.

Estudo realizado em três capitais brasileiras com jovens de ambos os sexos, a fim de observar a sequência temporal entre abandono escolar e gravidez na adolescência, mostrou que, entre os mais pobres, registra-se uma maior proporção de moças que abandonaram os estudos à época ou depois da gravidez, informação também obtida entre rapazes que, na adolescência, engravidaram suas parceiras ${ }^{15}$.

No âmbito internacional, estudos recentes na América Latina documentaram que, no México, a maternidade na adolescência reduz os anos de escolaridade e as horas trabalhadas; em outros casos, como no Chile, a maternidade na adolescência diminui a probabilidade de terminar o ensino médio e prosseguir os estudos pós-secundários ${ }^{35,36}$.

De um modo geral, em razão de a gravidez estar relacionada a um contexto de vulnerabilidade social, é necessário considerar que os resultados do presente estudo, assim como os de vários outros, evidenciam que sua frequência ocorre em maior intensidade entre aqueles que estão inseridos em ambientes marcados por oportunidades restritas e poucas opções de vida. A explicação para isso pode ter como base a observação de que municípios com baixos índices de desenvolvimento humano e com maiores incidências de pobreza são aqueles com maior número de gravidez na adolescência. Se a maternidade na adolescência reduz a escolaridade e a oportunidade laboral da mãe, e se essa realidade é muito comum nos lares mais pobres, então se produz um dos mais reconhecidos ciclos de reprodução intergeracional da pobreza: jovens mais pobres são mães adolescentes, e a maternidade precoce encolhe a sua escolaridade e suas oportunidades futuras, mantendo mães e filhos em situação de pobreza.

Não obstante, compreende-se que a gestação na adolescência não constitui um problema em si, mas ao contexto de iniquidades que a produz e reproduz, sendo fundamental que as políticas sociais atentem para as necessidades e demandas específicas dessa população, na construção de estratégias que atuem nos determinantes sociais e na redução de vulnerabilidades que podem ser desfavoráveis para a qualidade de vida dessa população ${ }^{37}$.

Mesmo com as contribuições realizadas pelo estudo, a não investigação sobre a aptidão de idade-série no momento da entrada de jovens na escola e o não questionamento sobre a realização do exame para a universidade e sobre a quantidade de tentativas constituem limitações que devem ser consideradas, uma vez que não permite afirmar que a incompatibilidade idade-série está presente desde a origem da vida estudantil dessas jovens e se elas tentaram prosseguir o caminho acadêmico, o que é comum às filhas de classe média. Todavia, o acesso ao ensino superior, tanto por adolescentes de classe média como por aquelas das classes menos favorecidas, deve ser uma opção viável se essa for a opção de qualquer jovem, e não um privilégio de grupos em vantagem social.

Nesse sentido, conclui-se que a condição social da adolescente é um fator com maior força para o abandono escolar do que a própria gravidez, visto que esta se apresentou apenas como um evento constituinte do ciclo. Assim, reafirma-se a importância de manter a jovem mãe na escola no sentido de interromper o ciclo vicioso: pobreza - gravidez - abandono escolar - pobreza.

Diante disso, cabe ao sistema escolar lidar com as heterogeneidades juvenis, adotando política educacional que oriente as jovens quanto às suas escolhas relativas à sexualidade, e, em conjunto com a família, estimular a permanência delas na escola e o seguimento dos planos de vida. Além disso, é preciso traçar estratégias voltadas às jovens evadidas, de modo a possibilitar o seu retorno ao ambiente escolar, oferecendo alternativas que englobem trabalho e/ou filhos.

Quanto à saúde, é fundamental a implementação de políticas públicas que vão muito além da abordagem meramente preventiva, mas que contemplem as reais necessidades das jovens, escutando-as e levando em consideração as particularidades socioeconômicas e comportamentais da população em questão, promovendo intervenções que tenham como ponto de partida a educação sexual e reprodutiva, de modo que as escolhas das adolescentes sejam conscientes e fortaleçam o futuro delas.

Além disso, a concretização das ações do Programa Saúde na Escola (PSE), que tem por finalidade realizar um trabalho intersetorial entre educação e saúde, constitui uma estratégia importante na abordagem da situação de saúde da população estudada e dos problemas que a acomete, tais como o trabalhado neste artigo. 


\section{REFERÊNCIAS}

1. World Health Organization. Adolescent health. [Internet]. Geneva; 2017 [citado em 2017 mar 24]. (WHO Health Topics). Disponível em: http:// www.who.int/topics/adolescent_health/en/.

2. Martinez EZ, Roza DL, Caccia-Bava MC, Achcar JA, Dal-Fabbro AL. Gravidez na adolescência e características socioeconômicas dos municípios do Estado de São Paulo, Brasil: análise espacial. Cad Saude Publica. 2011;27(5):855-67. http://dx.doi.org/10.1590/S0102-311X2011000500004. PMid:21655837.

3. Sousa-Mata AL, Araújo-Lemos C, Lira-Ferreira C, Pereira-Braga L, ChavesMaia EM. Fatores de risco na repetição de gravidez na adolescência. Rev Colombiana de Psicologia. 2009;18(2):167-75.

4. World Health Organization. 2012. Early marriages, adolescent and young pregnancies. Provisional agenda item 13.4. In: Proceedings $65^{\circ}$ Word Health Assembly. 2012 mai 21-26; Geneva. Geneva: WHO; 2012 [citado em 2017 mar 24]. Disponível em: <http://apps.who.int/gb/ebwha/pdf_files/WHA65/ A65_13-en.pdf>.

5. World Health Organization. Adolescent pregnancy [Internet]. Geneva: WHO; 2014 [citado em 2017 mar 24]. (Fact sheet, 364). Disponível em: http://www.who.int/mediacentre/factsheets/fs364/en/

6. Ministério da Saúde. DATASUS. Informações em Saúde. Nascidos vivos Brasil [Internet]. 2012a. [citado em 2017 mar. 24]. Disponível em: http:// tabnet.datasus.gov.br/cgi/tabcgi.exe?sinasc/cnv/nvuf.def

7. Ministério da Saúde. DATASUS. Informações em Saúde. Nascidos vivos -Piauí [Internet]. 2012b. [citado em 2017 mar 24]. Disponível em: http:// tabnet.datasus.gov.br/cgi/tabcgi.exe?sinasc/cnv/nvpi.def

8. Oliveira-Monteiro NR, Negri M, Fernandes AO, Nascimento JOG, Montesano FT. Gravidez e maternidade de adolescentes: fatores de risco e de proteção. Rev Bras Crescimento Desenvol Hum. 2011;21(2):198-209.

9. Santos JO, Silva CFS, Petenão E, Soster FCB, Berard MB, Silva SR. Perfil das adolescentes com reincidência de gravidez assistidas no setor público de Indaiatuba (SP). Rev Inst Cienc Saude. 2009;27(2):115-21.

10. Almeida MCC, Aquino EML, Barros AP. School trajectory and teenage pregnancy in three Brazilian state capitals. Cad Saude Publica. 2006;22(7):142130. http://dx.doi.org/10.1590/S0102-311X2006000700005.

11. Sabroza AR, Leal MC, Gama SGN, Costa JV. Perfil sócio-demográfico e psicossocial de puérperas adolescentes do município do Rio de Janeiro, Brasil - 1999-2001. Cad Saude Publica. 2004;20(1 Supl):S112-120.

12. Instituto Brasileiro de Geografia e Estatística. Coordenação de Trabalho e Rendimento. Pesquisa Nacional por Amostra de Domicílios: síntese de indicadores 2014/IBGE. Rio de Janeiro: IBGE; 2015.

13. WORLD BANK. Relatório Annual de 2016 [Internet] Washington; 2016 [citado em 2017 jun 29] Disponível em: https://www.google.com.br/sear ch?ei=THZGW9TjDqvy5gKCgpugCg\&q=WORLD+BANK+Relat\%C3\% B3rio + Annual+de+2016\&oq $=$ WORLD + BANK+Relat $\%$ C3\%B3rio + Annu al+de+2016\&gs_l=psy-ab.3...14944.16748.0.17271.3.3.0.0.0.0.750.1288.51j1.2.0...0...1.1.64.psy-ab..1.0.0....0.Y6udNeTX7aw

14. Rosenberg M, Pettifor A, Miller WC, Thirumurthy H, Emch M, Afolabi SA, et al. Relationship between school dropout and teen pregnancy among rural South African Young women. Int J Epidemiol. 2015;44(3):928-36. http://dx.doi.org/10.1093/ije/dyv007. PMid:25716986.

15. Almeida MCC. Gravidez na adolescência e escolaridade: um estudo em três capitais brasileiras [tese] Bahia: Universidade Federal da Bahia. Instituto de Saúde Coletiva; 2008.
16. Chalem E, Mitsuhiro SS, Ferri CP, Barros MCM, Guinsburg R, Laranjeira R. Gravidez na adolescência: perfil sociodemográfico e comportamental de uma população da periferia de São Paulo, Brasil. Cad Saude Publica. 2007;23(1):177-86. http://dx.doi.org/10.1590/S0102-311X2007000100019. PMid:17187116.

17. Taborda JA, Silva FC, Ulbricht L, Neves EB. Consequências da gravidez na adolescência para as meninas considerando-se as diferenças socioeconômicas entre elas. Cad Saude Colet. 2014;22(1):16-24. http://dx.doi.org/10.1590/1414462X201400010004.

18. Crittenden CP, Boris NW, Rice JC, Taylor CA, Olds DL. The role of mental health factors, behavioral factors, and past experiences in the prediction of rapid repeat pregnancy in adolescence. J Adolesc Health. 2009;44(1):25-32. http://dx.doi.org/10.1016/j.jadohealth.2008.06.003. PMid:19101455.

19. Gomes KRO, Speizer IS, Gomes FM, Oliveira DC, Moura LN. Who are the pregnant adolescents in the poorest state capital of Brazil? Public Health Nurs. 2008;25(4):319-26. http://dx.doi.org/10.1111/j.1525-1446.2008.00712.x. PMid:18666937.

20. World Health Organization. Adolescent pregnancy: issues in adolescent health and development [Internet]. Geneva; 2004 [citado em 2017 jun 1]. Disponível em: http://apps.who.int/iris/bitstream/handle/10665/42903/9241591455_eng. pdf;jsessionid=B6E4D335460B0918CBBD786E920CE609? sequence $=1$

21. World Health Organization. Child and adolescent health development [Internet] Geneva; 2001 [citado em 2017 jun 1]. Disponível em: http:// www.who.int/child-adolescent-health/OVERVIEW/AHD/adh-over.htm

22. WORLD BANK. World Bank list of economies [Internet]. 2008 [citado em 2009 apr 21]. Disponível em: http://siteresources.worldbank.org/ DATASTATISTICS/Resources/CLASS.XLS

23. Nery IS, Mendonça RC, Gomes IS, Fernandes AC, Oliveira DC. Recorrência da gravidez em adolescentes de Teresina, PI, Brasil. Rev Bras Enferm. 2011;64(1):31-7. http://dx.doi.org/10.1590/S0034-71672011000100005. PMid:21468486.

24. Barnet B, Arroyo C, Devoe M, Duggan AK. Reduced school dropout rates among adolescents mothers receiving school-bades prenatal care. Arch Pediatr Adolesc Med. 2004;158(3):262-8. http://dx.doi.org/10.1001/ archpedi.158.3.262. PMid:14993086

25. Grant MJ, Hallman KK. Pregnancy-related school dropout and prior school performance in Kwa-Zulu-Natal, South Africa. Stud Fam Plann. 2008;39(4):369-82. http://dx.doi.org/10.1111/j.1728-4465.2008.00181.x. PMid:19248721.

26. Almeida MC, Aquino EM. The role of education level in the intergenerational pattern of adolescente pregnancy in Brazil. Int Perspect Sex Reprod Health. 2009;35(3):139-46. http://dx.doi.org/10.1363/3513909. PMid:19805019.

27. Michelazzo D, Yazlle MEHD, Mendes MC, Patta MC, Rocha JSY, Moura MD. Indicadores sociais de grávidas adolescentes: estudo caso-controle. Rev Bras Ginecol Obstet. 2004;26(8):633-9. http://dx.doi.org/10.1590/ S0100-72032004000800007.

28. Nascimento MC, Xavier PF, Passos De Sá RD. Adolescentes grávidas: a vivência no âmbito familiar e social. Adoles Saude. 2011;8(4):41-7.

29. Yazlle MEHD, Mendes MC, Patta MC, Rocha JSY, Azevedo GD, Marcolin AC. A adolescente grávida: alguns indicadores sociais. Rev Bras Ginecol Obstet. 2002;24(9):609-14. http://dx.doi.org/10.1590/S0100-72032002000900007. 
30. Kassar SB, Lima MC, Albuquerque MFM, Barbieri MA, Gurgel RQ. Comparações das condições socioeconômicas e reprodutivas entre mães adolescentes e adultas jovens em três maternidades públicas de Maceió, Brasil. Rev Bras Saude Mater Infant. 2006;6(4):397-403. http://dx.doi. org/10.1590/S1519-38292006000400006.

31. World Health Organization. Adolescent pregnancy: a culturally complex issue. Geneva: Bull World Heath Organ; 2009.

32. Bruno ZV, Feitosa FEL, Silveira KP, Morais IQ, Bezerra MF. Reincidência de gravidez em adolescentes. Rev Bras Ginecol Obstet. 2009;31(10):480-4. http://dx.doi.org/10.1590/S0100-72032009001000002. PMid:19942994.

33. Silva KS, Rozenberg R, Bonan C, Chuva VC, Costa SF, Gomes MA. Gravidez recorrente na adolescência e vulnerabilidade social no Rio de Janeiro (RJ, Brasil): uma análise de dados do Sistema de Nascidos Vivos. Cien Saude Colet. 2011;16(5):2485-93. http://dx.doi.org/10.1590/S141381232011000500018 .
34. Moura LNB, Gomes KRO, Sousa CRO, Maranhão TA. Multiparidade entre adolescentes e jovens e fatores de risco em Teresina/Piauí. Adoles Saude. 2014;11(3):51-62.

35. Aceo-Gómez E, Campos-Váquez RM. Teenage pregnancy in Mexico: evolution and consequences. 2011. [citado em 2009 apr 21]. Disponível em: https://scielo.conicyt.cl/pdf/laje/v51n1/art04.pdf.

36. Kruger D, Berthelon M. Education consequences of adolescent motherhood in Chile. Washington: Banco Mundial; 2012.

37. Ferreira RA, Ferriani MGC, Mello DF, Carvalho IP, Oliveira LA. Análise espacial da vulnerabilidade social da gravidez na adolescência. Cad Saúde Pública. 2012; 28(2):313-323.

Recebido em: Dez. 18, 2017 Aceito em: Jun. 19, 2018 